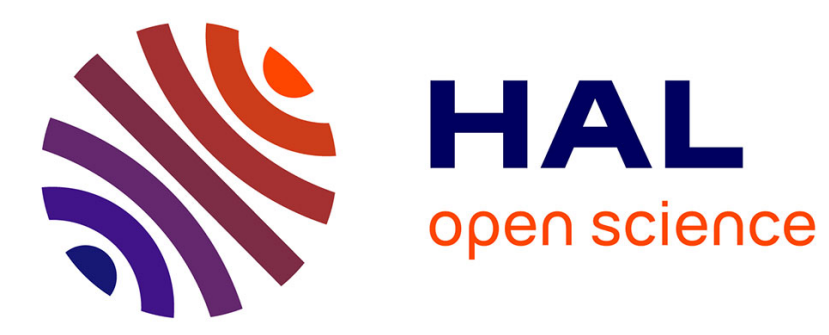

\title{
Diagnostic d'un faisceau de plasma autour d'un corps positif et application à la sonde de sayers
}

\author{
G. Fournier
}

\section{To cite this version:}

G. Fournier. Diagnostic d'un faisceau de plasma autour d'un corps positif et application à la sonde de sayers. Revue de Physique Appliquée, 1973, 8 (4), pp.341-350. 10.1051/rphysap:0197300804034100 . jpa-00243693

\section{HAL Id: jpa-00243693 https://hal.science/jpa-00243693}

Submitted on 1 Jan 1973

HAL is a multi-disciplinary open access archive for the deposit and dissemination of scientific research documents, whether they are published or not. The documents may come from teaching and research institutions in France or abroad, or from public or private research centers.
L'archive ouverte pluridisciplinaire HAL, est destinée au dépôt et à la diffusion de documents scientifiques de niveau recherche, publiés ou non, émanant des établissements d'enseignement et de recherche français ou étrangers, des laboratoires publics ou privés. 


\title{
DIAGNOSTIC D'UN FAISCEAU DE PLASMA AUTOUR D'UN CORPS POSITIF ET APPLICATION A LA SONDE DE SAYERS
}

\author{
G. FOURNIER \\ ONERA, 92320 Châtillon, France
}

(Reçu le 14 février 1973)

\begin{abstract}
Résumé. - On analyse la constitution des gaines autour d'un corps polarisé positivement en vol ionosphérique. Ces résultats permettent d'expliquer les difficultés de mise en œuvre de la sonde de Sayers destinée à mesurer la densité électronique.
\end{abstract}

Abstract. - Space-charge sheaths of positively biased bodies in ionospheric flight are analyzed. These results explain the difficulties encountered in using the Sayers' electron-density probe.

1. Introduction. - Une sonde, destinée à mesurer la densité électronique dans l'ionosphère et conçue par le Professeur Sayers [1], a été utilisée à bord du satellite FR-1. Les résultats obtenus [2] n'ont pu jusqu'à présent être interprétés, ce qui nécessite d'approfondir l'étude du comportement de cette sonde.

La sonde de Sayers est constituée de deux grilles parallèles ressemblant à de petites raquettes. L'ensemble est polarisé à une tension lentement variable depuis les valeurs négatives jusqu'aux valeurs positives par rapport au potentiel plasma. Entre les grilles est appliquée une tension alternative de pulsation constante $\omega$ supérieure à la pulsation plasma $\omega_{\mathrm{p}}$. La mesure du courant entre les grilles fournit la capacité du système. Si cette capacité en absence de plasma est $C_{0}$, elle devient, en présence d'une densité électronique uniforme, égale à :

$$
C=C_{0}\left(1-\omega_{\mathrm{p}}^{2} / \omega^{2}\right),
$$

d'après l'équation d'Appleton-Hartree, où les effets de l'agitation thermique, des collisions et du champ magnétique sont négligés. La valeur de

$$
\omega_{\mathrm{p}}^{2}=n e^{2} / \varepsilon_{0} m
$$

où $e$ est la charge de l'électron, $m$ sa masse et $\varepsilon_{0}$ la permittivité du vide, permet de déterminer la densité électronique $n$.

Pour des raisons pratiques, il est utile d'étalonner le dispositif en cours d'expérience. La valeur de référence $C_{0}$ correspondant à l'absence d'électrons est aisément obtenue : la distance inter-grilles étant de l'ordre de la longueur de Debye, les gaines vides d'électrons occupent pour des polarisations suffisamment négatives tout l'espace déterminant la capacité.

Il reste à repérer le potentiel plasma pour lequel on suppose que la densité électronique, autour et dans la sonde, est homogène et égale à celle du plasma ambiant, les gaines entourant les électrodes disparaissant pour ce potentiel : cette supposition correspond à l'hypothèse suivant laquelle on néglige les effets d'absorption de particules dus aux grilles (leur transparence est d'environ $75 \%$ en général). D'après ce qui précède, le courant alternatif décroît depuis les potentiels négatifs jusqu'au potentiel plasma. Si l'on excepte l'interprétation erronée des premiers travaux [1], Sayers et al. [3] se bornent à affirmer qu'au-delà du potentiel plasma, la vitesse moyenne des particules croît, donc leur densité décroît dans la région de la sonde. Le courant alternatif mesuré aux bornes du condensateur passerait alors par un minimum au potentiel plasma.

Ce n'est malheureusement pas ce que donnent de nombreuses expériences. Sayers lui-même [4] est conscient qu'un effet de concentration d'électrons dû à la convection vers la sonde peut éventuellement compenser l'effet de l'augmentation de vitesse et se traduire par une diminution du courant au-delà du potentiel plasma. Un effort de compréhension plus poussé a été entrepris avec succès par Goodall [5]. Se basant sur le fait que la vitesse moyenne des électrons évolue comme $\sqrt{\varphi}$ ( $\varphi$ étant le potentiel par rapport au plasma ambiant réduit à l'énergie thermique des électrons) et que le courant reçu par une sonde électrostatique ordinaire évolue en $\varphi^{0}, \varphi^{1 / 2}$ ou $\varphi$ respectivement pour un plan, un petit cylindre ou une petite sphère, il en conclut que la densité évolue en $\varphi^{-1 / 2}, \varphi^{0}$ ou $\varphi^{1 / 2}$ dans une géométrie respectivement plane, cylindrique ou sphérique. Comme il s'agit là des fondements du fonctionnement de la sonde de Sayers, nous avons jugé qu'il était nécessaire d'éclaircir quelque peu ces notions. 
Nous venons de raisonner sur le potentiel de la sonde par rapport au plasma. Mais le seul paramètre bien connu est le potentiel de polarisation de la sonde par rapport au corps du satellite ou de la fusée. Pour être certain de pouvoir polariser positivement la sonde par rapport au plasma, des cathodes émissives ont été montées sur le satellite FR-1. Cette question des polarisations est reprise dans la deuxième partie des considérations fondamentales à cause de son évidente importance.

Nous proposons ensuite deux moyens de diagnostic pour étudier le plasma dans et au voisinage de la sonde de Sayers. Des indications sur la densité électronique sont fournies par une simple sonde cylindrique dont on mesure le potentiel flottant. Quant au potentiel local du plasma, il est obtenu à l'aide d'une petite sonde émissive. Une interprétation de ces mesures à l'aide de calculs simples est proposée puis testée sur une expérience préliminaire dans laquelle on étudie le plasma autour d'une plaque mince puis autour d'une grille. Ces sondes sont ensuite utilisées pour analyser le plasma voisin de la sonde de Sayers.

2. Considérations fondamentales. -2.1 Densité ÉLECTRONIQUE DANS UNE RÉGION POSITIVE PAR RAPPORT AU PLASMA AMBIANT. - Soit une population d'électrons maxwellienne de densité $n_{\infty}$ et de température $T_{\mathrm{e}}$. Dans l'espace hodographe cartésien, leur fonction de distribution $f$ pour les variables de vitesses $w_{x}, w_{y}, w_{z}$ est donnée par :

$f=n_{\infty}\left(m / 2 \pi k T_{\mathrm{e}}\right)^{3 / 2} \exp \left[-m\left(w_{x}^{2}+w_{y}^{2}+w_{z}^{2}\right) / 2 k T_{\mathrm{e}}\right]$,

où $k$ est la constante de Boltzmann. Cette hypothèse correspond au plasma vu par un engin ionosphérique dont la vitesse est toujours très inférieure à la vitesse thermique des électrons.

Nous nous proposons d'étudier les propriétés de ces électrons dans une région dont le potentiel diffère du potentiel ambiant de référence et qui est suffisamment petite pour négliger les collisions. Les effets du champ magnétique sont également négligés et nous n'étudions que le régime stationnaire. Les calculs développés dans l'appendice I donnent une borne supérieure de la densité électronique dans une région à une, deux ou trois dimensions où le potentiel est positif.

Les expressions (A.7), (A.9) et (A.11) sont représentées graphiquement sur la figure 1. Pour $\varphi \rightarrow \infty$ ( $\varphi$ est défini par la formule A.1), elles s'accordent bien avec les considérations qualitatives de Goodall [5]. D'après les formules (A.7) et (A.9), la densité électronique passe par un maximum au potentiel plasma mais la formule (A.11) indique qu'elle peut continuer à croître au-delà du potentiel plasma. Goodall [5] fait allusion à des calculs de potentiels sans en donner la référence mais on conçoit bien que les sondes de Sayers habituelles qui ont leurs plus grandes dimen- sions de l'ordre de quelques longueurs de Debye seulement puissent donner des équipotentielles presque sphériques; on se rapprocherait alors d'autant plus

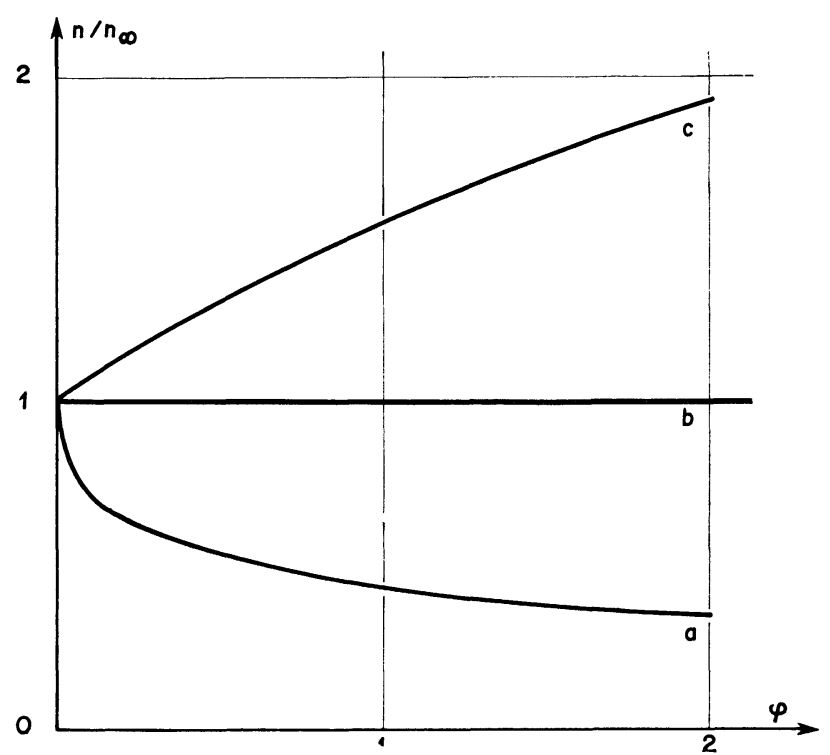

Fig. 1. - Borne supérieure de la densité électronique dans un potentiel positif : $a$ ) cas monodimensionnel, $b$ ) cas bidimensionnel, c) cas tridimensionnel.

de la borne supérieure (A.11) que l'absorption des grilles est plus faible. L'amélioration qui consiste à allonger les raquettes [5] est donc importante. Cependant, dans le pire des cas où la densité suivrait l'expression de la borne supérieure (A.11), il existe tout de même au potentiel plasma un changement de pente qui n'apparaît d'ailleurs bien qu'en coordonnées semi-logarithmiques (Fig. 2).

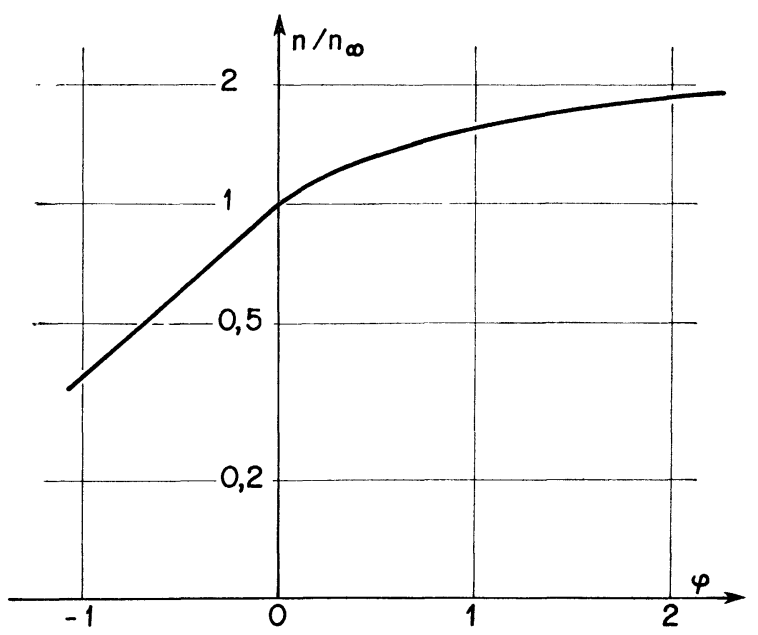

FIG. 2. - Borne supérieure de la densité électronique dans une sonde de Sayers.

Par ailleurs, les formules (A.7), (A.9) et (A.11) permettent d'estimer l'étendue des gaines des corps positifs en écoulement mésothermique. Un plasma 
de vitesse moyenne $v$ par rapport au corps étudié est dit mésothermique si

$$
\left(2 k T_{\mathrm{i}} / M\right)^{1 / 2} \ll v \ll\left(2 k T_{\mathrm{e}} / m\right)^{1 / 2} ;
$$

$T_{\mathrm{i}}$ est la température des ions et $M$ leur masse. Cette relation est bien vérifiée pour le vol ionosphérique d'un satellite. Comme généralement $T_{\mathrm{e}}$ et $T_{\mathrm{i}}$ sont du même ordre de grandeur, des potentiels agissant fortement sur les électrons n'ont qu'un effet négligeable sur les ions. C'est pourquoi le mécanisme classique de formation de gaine, essentiellement basé sur la répulsion d'une espèce, ne joue pas pour les corps positifs dans un plasma mésothermique [8].

Dans les géométries à une et à deux dimensions, les relations (A.7) et (A.9) montrent que $n<n_{\infty}$ (strictement, en présence d'un corps absorbant positif); la condition de raccordement avec le milieu ambiant, qui exige la quasi-neutralité, impose donc que la densité ionique soit inférieure à $n_{\infty}$. La longueur caractérisant l'étendue du champ n'est plus la longueur de Debye ordinaire

$$
h=\left(k T_{\mathrm{e}} / \varepsilon_{0} e^{2} n_{\infty}\right)^{1 / 2},
$$

mais

$$
h^{\prime}=\left(M v^{2} / 2 \varepsilon_{0} e^{2} n_{\infty}\right)^{1 / 2}
$$

où l'énergie des électrons a été remplacée par l'énergie dirigée des ions. En pratique $h^{\prime} \approx 10 h$. Les résultats d'un calcul numérique avec un cylindre sont donnés dans les références [7], [8].

Par contre, si l'on considère les trois dimensions, la relation (A.11) montre qu'on peut avoir un excès d'électrons $\left(n>n_{\infty}\right)$. D'après les calculs numériques de Laframboise [6], ceci ne se produit en fait que pour des sphères de rayon $\leqslant h$ en ordre de grandeur. On peut ainsi résumer ces considérations qualitatives : pour un corps positif dont la plus grande dimension est inférieure ou égale à $h$ (en ordre de grandeur), il se peut que les perturbations dues à son potentiel soient d'étendue limitée ; si la plus grande dimension est supérieure à $10 h$ (en ordre de grandeur), les perturbations de potentiel doivent s'étendre à plusieurs fois $10 h$.

2.2 POTENTIEL DE LA SONDE PAR RAPPORT AU PLASMA. - Appelons $\Delta \varphi$ le potentiel de la sonde par rapport au satellite (en valeurs réduites), $\varphi$ demeurant le potentiel de la sonde par rapport au plasma ambiant. En absence de surface émissive, les courants à considérer sont, en première approximation, les suivants :

- courant d'ions sur satellite : proportionnel à $v S / 4$,

— courant d'électrons sur satellite : proportionnel à

$$
\frac{1}{4} \sqrt{\frac{8 k T_{\mathrm{e}}}{\pi m}} S \exp (\varphi-\Delta \varphi),
$$

- courant d'ions sur sonde : proportionnel à $v s / 4$,
- courant d'électrons sur sonde : proportionnel à

$$
\frac{1}{4} \sqrt{\frac{8 k T_{\mathrm{e}}}{\pi m}} s(1+\varphi),
$$

$S$ est la surface du satellite (supposé sphérique) et $s$ est la surface de collection de la sonde : l'évolution de son courant en $(1+\varphi)$ correspond au cas d'une petite sphère. En pratique :

$$
v /\left(8 k T_{\mathrm{e}} / \pi m\right)^{1 / 2} \approx 5 \times 10^{-2} .
$$

En écrivant que la somme des courants sur la sonde et le satellite est nulle, on obtient pour des rapports $S / s$ suffisamment grands $\left(\approx 10^{3}\right)$ :

$$
\varphi=\Delta \varphi+\text { Cte }
$$

(la constante qui est d'environ -3 est le potentiel flottant du satellite).

Alors le potentiel de la sonde par rapport au plasma évolue linéairement avec son potentiel de polarisation. Mais pour des rapports $S / s$ plus faibles, comme dans le cas du satellite FR-1 où la majeure partie de la surface est couverte de cellules solaires isolantes, il n'y a plus de relation linéaire entre $\varphi$ et $\Delta \varphi$ et il peut même être impossible d'atteindre $\varphi=0$, si la sonde collecte au potentiel plasma un courant d'électrons supérieur au courant d'ions reçu par le satellite. Il est alors nécessaire d'utiliser des surfaces émissives d'électrons.

En présence d'une surface émissive, le courant d'électrons reçu par la sonde, en $(1+\varphi)$, est compensé par un courant d'émission en $(\Delta \varphi-\varphi)^{3 / 2}$ : ceci peut conduire à une relation non linéaire où $\Delta \varphi$ croît plus vite que $\varphi$. Comme il a été dit que l'influence du courant d'émission peut apparaître avant le potentiel plasma, celui-ci ne sera même plus caractérisé comme sur la figure 2. D'autre part, il faudra examiner si les électrons introduits ne perturbent pas le plasma au voisinage de la sonde.

3. Moyens de diagnostic. - Pour mesurer localement la densité électronique et le potentiel au voisinage d'un corps positif, nous utilisons des sondes technologiquement simples, mais leur mode d'utilisation et l'exploitation de leurs données nécessitent quelques commentaires. Ce chapitre ne concerne qu'un plasma mésothermique où la relation (4) est satisfaite.

3.1 ETUdE DE LA DENSITÉ ÉLECTRONIQUE. - Elle est faite à l'aide d'une sonde auxiliaire cylindrique en molybdène de $1 \mathrm{~cm}$ de long et $0,5 \mathrm{~mm}$ de diamètre dont on mesure le potentiel flottant.

Tant que les potentiels de la sonde auxiliaire sont peu importants,

$$
|\varphi| \ll M v^{2} / 2 k T_{\mathrm{e}},
$$

soit en pratique quelques volts, le courant d'ions qu'elle collecte est constant car son axe est perpendiculaire au faisceau. Les variations de potentiel flottant 
sont donc directement liées aux phénomènes électroniques.

Attendu que les dimensions de la sonde auxiliaire sont très petites devant celles des régions étudiées, et que le courant reçu est imposé à une valeur faible (égale au courant d'ions), nous négligerons les effets d'absorption d'électrons par cette sonde sur le plasma environnant.

Ce plasma environnant n'est donc modifié qu'à cause du potentiel de la sonde auxiliaire. Tant que celui-ci est faible et négatif, son rayon de perturbation est de l'ordre de la longueur de Debye $h$ : ainsi les informations données par cette sonde concernent globalement une région d'étendue de l'ordre de $h$.

Soit $\varphi_{\mathrm{s}}$ le potentiel réduit de la sonde auxiliaire par rapport au plasma ambiant de référence; soit $\varphi$ le potentiel du plasma à proximité de la sonde auxiliaire mais suffisamment loin d'elle pour ne pas être modifié par elle. Cette définition a un sens car nous avons vu que la distance de perturbation de la sonde auxiliaire flottante est de l'ordre de $h$ alors que les perturbations dues à des corps positifs sont de l'ordre de $10 h$. Dans l'appendice II nous rattachons $\varphi_{\mathrm{s}}$ aux paramètres caractérisant le plasma local (potentiel $\varphi$ et densité $n$ ). Ces calculs (voir Fig. 3) font apparaître les conclusions suivantes :

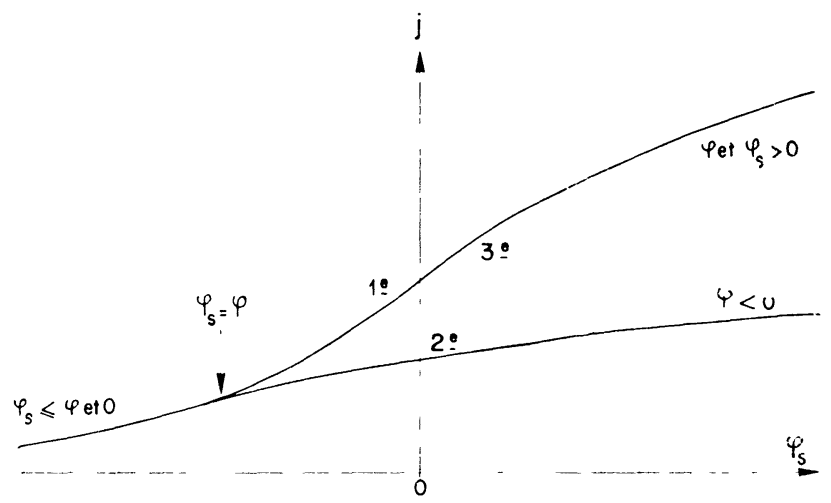

Fig. 3. - Courant de sonde auxiliaire $j$ en fonction de son potentiel $\varphi_{\mathrm{s}}$ suivant les trois équations (A.16).

- On aura toujours $\varphi_{\mathrm{s}} \geqslant \varphi_{\mathrm{s} \infty}$ tant que le courant d'ions ne sera pas modifié. $\varphi_{\mathrm{s}}<\varphi_{\mathrm{s} \infty}$ est caractéristique d'un effet de sillage ;

- pour $\varphi \ll 0, \varphi_{\text {s }}$ peut devenir fortement positif. Compte tenu de ce que la sonde apporte alors de fortes perturbations, la relation (A.18) permet d'avoir une valeur approximative de $\varphi$ qui concerne une région étendue (par rapport à la longueur de Debye) ;

- pour $\varphi_{\mathrm{s}}<\varphi$, la sonde est apte à déceler les variations de densité locale dues à des effets d'absorption mais pas les variations dues à des effets balistiques. Elle fournira donc une borne supérieure de la densité locale

$$
n \leqslant n_{\infty} \exp \left(\varphi_{\mathrm{s} \infty}-\varphi_{\mathrm{s}}\right)
$$

en géométrie à deux dimensions

$n \leqslant n_{\infty}\left(\exp \varphi \operatorname{erf} c \sqrt{\varphi}+\frac{2}{\sqrt{\pi}} \cdot \sqrt{\varphi}\right) \exp \left(\varphi_{\mathrm{s} \infty}-\varphi_{\mathrm{s}}\right)$

en géométrie à trois dimensions ; cette dernière information est d'un intérêt limité puisqu'elle ne permet pas de dire si $n$ est supérieur ou inférieur à $n_{\infty}$ sauf au potentiel plasma. Par contre la relation (9) est importante car elle permet de vérifier si la densité au sein d'une sonde de Sayers (de type allongé) est bien égale à la densité ambiante.

3.2 Mesure locale du POTENTIEL. - Elle est obtenue à l'aide d'un filament enroulé de $1 \mathrm{~mm}$ de long et quelques dixièmes de $\mathrm{mm}$ de diamètre, chauffé à blanc, dont on mesure le potentiel flottant à l'extrémité négative. La tension de chauffage est de $5 \mathrm{~V}$. Les caractéristiques de cette sonde émissive ont l'allure de celle représentée sur la figure 4 . Vu la valeur relativement élevée de la tension de chauffage, la plus grande partie de la sonde reste positive par rapport au plasma : le courant d'électrons qu'elle reçoit est proportionnel à la tension (comme le courant de saturation d'une petite sonde sphérique). Le courant d'électrons émis est proportionnel à la surface de la partie émissive du filament.

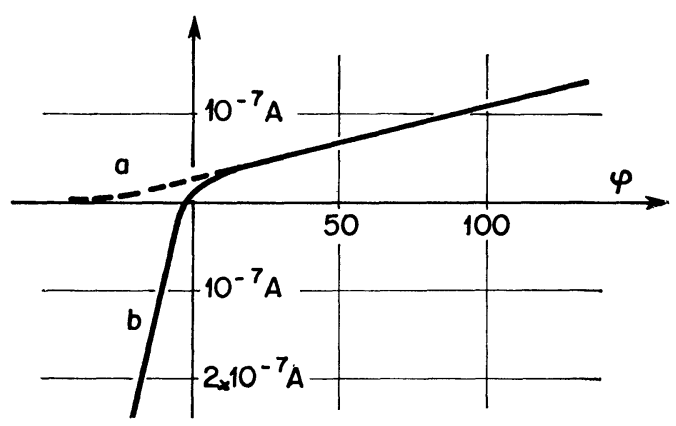

Fig. 4. - Caractéristique de la sonde émissive : a) froide, b) émissive.

Les perturbations apportées par ce type de sonde sont ici réduites au mieux. Son potentiel, bien qu'en partie positif, ne s'étend pas loin car la sonde est de petite taille par rapport à $h$ (voir $\S 2.1)$. La population électronique prélevée est bien compensée par celle produite puisque les courants sont égaux (potentiel flottant) et les températures voisines $(\approx 1000 \mathrm{~K})$.

Le potentiel flottant $\varphi_{\mathrm{s}}$ de l'extrémité négative de sonde suit l'évolution du potentiel $\varphi$ du plasma environnant tant que le courant reçu est constant. Lorsque ce dernier est modifié pour une raison quelconque, on peut effectuer la correction suivante. La référence de courant reçu est obtenue en prolongeant la partie rectiligne correspondant au courant reçu jusqu'au potentiel flottant : la différence de ces valeurs de référence est $\Delta i$. La variation de potentiel correspondant au $\Delta i$ reporté sur la caractéristique d'émission est la 
correction cherchée. Vu la forte valeur de la pente de la caractéristique d'émission, il est souvent inutile d'effectuer cette correction tant qu'on explore des régions à $\varphi>0$ où l'absorption est faible.

Avant de les appliquer à la sonde de Sayers, nous avons testé ces moyens de diagnostic sur des expériences préliminaires.

4. Dispositif expérimental. - Nous avons utilisé le simulateur de vol ionosphérique de l'ONERA. Il s'agit d'une source de plasma neutralisé à basse énergie fonctionnant dans un caisson à vide poussé de grandes dimensions [9].

Dans les expériences suivantes, les conditions étaient :

$$
\left.\begin{array}{l}
T_{\mathrm{e}}: 550-900 \mathrm{~K} \\
n: 1,5-2,3 \times 10^{5} \mathrm{~cm}^{-3}
\end{array}\right\} h \approx 0,5 \mathrm{~cm} .
$$

Pression : $1-1,4 \times 10^{-4} \mathrm{~N} / \mathrm{m}^{2}$.

Ions d'argon de $20 \mathrm{eV}(v=10 \mathrm{~km} / \mathrm{s})$.

Des chariots permettent de déplacer des sondes dans deux dimensions. Tous les objets en contact avec le plasma étudié ont été soigneusement décontaminés par bombardement ionique ou par chauffage à haute température [9].

Courants et tensions sont mesurés à l'aide d'un multimètre à très forte impédance (Keithley $600 \mathrm{~B}$ ). La source et les appareils de mesure sont suffisamment stables pour suivre des variations de potentiel de l'ordre de quelques $\mathrm{mV}$.

5. Expériences préliminaires. - Nous avons étudié le plasma autour d'une plaque plane en acier inoxydable de $500 \times 100 \times 1 \mathrm{~mm}$ puis autour d'une grille plane de mêmes dimensions dont la transparence était de $70 \%$. La plaque ou la grille étaient parallèles au jet suivant leur dimension $100 \mathrm{~mm}$ et les sondes étaient déplacées perpendiculairement aux surfaces à 2 ou $3 \mathrm{~cm}$ en aval du bord d'attaque. Les conditions expérimentales étaient :

$$
T_{\mathrm{e}}=750 \mathrm{~K}, \quad n_{\infty}=1,5 \times 10^{5} \mathrm{~cm}^{-3}(h=5 \mathrm{~mm}) .
$$

5.1 RÉSULTATS OBTENUS AVEC LA SONDE AUXILIAIRE CYLINDRIQUE FLOTTANTE. - Les figures 5 et 6 , pour la grille et la plaque respectivement, représentent l'évolution du potentiel flottant de la sonde auxiliaire en fonction de la distance par rapport à la surface. Plusieurs conclusions se dégagent de ces figures.

1) Suivant une remarque déjà formulée [7], le potentiel du plasma de référence est indépendant de la polarisation de l'obstacle tant que celle-ci est négative mais il croît lorsqu'elle est positive (par rapport au plasma). Ce phénomène a constitué la première preuve expérimentale de l'extension des effets des corps positifs en écoulement mésothermique bien au-delà de quelques longueurs de Debye (plusieurs centaines).

2) Pour les polarisations d'obstacle négatives, le potentiel de la sonde flottante est constant au loin

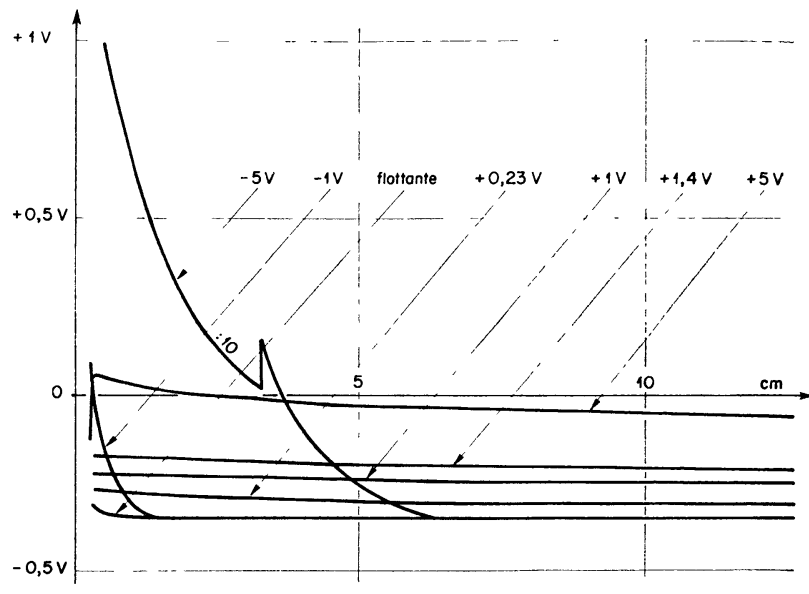

FIG. 5. - Potentiel de la sonde auxiliaire cylindrique flottante en fonction de sa distance à la grille pour cinq polarisations de celle-ci (il y a un changement d'échelle sur la courbe $-5 \mathrm{~V}$ ).

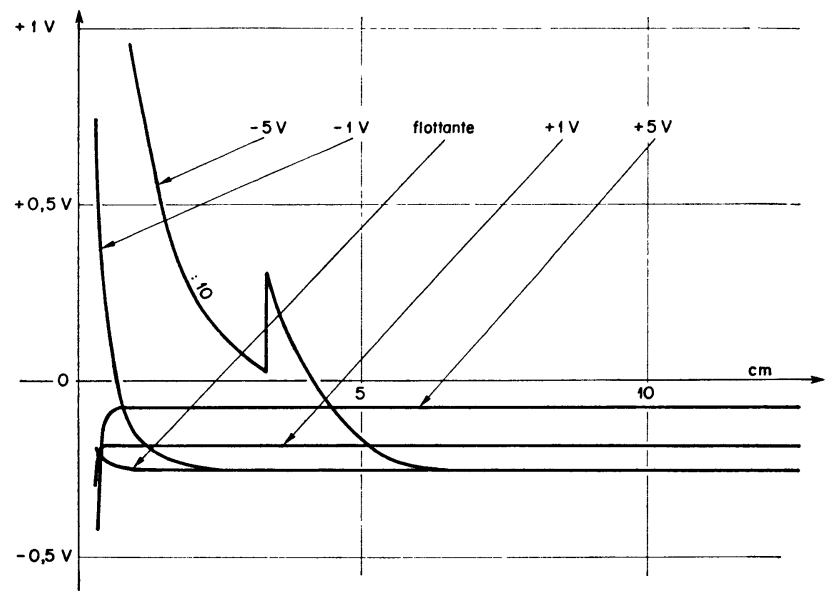

FIG. 6. - Potentiel flottant de la sonde auxiliaire cyclindrique flottante en fonction de sa distance à la plaque pour sept polarisations de celle-ci. La plaque à $+0,23 \mathrm{~V}$ (ou $+1,4 \mathrm{~V})$ reçoit le même courant d'électrons que la grille à $+1 \mathrm{~V}(\mathrm{ou}+5 \mathrm{~V})$. (Il y a un changement d'échelle sur la courbe $-5 \mathrm{~V}$.)

mais croît fortement au voisinage du corps négatif. Ce comportement paradoxal est conforme aux prévisions du paragraphe 3.1 . La relation (A.18) permet d'estimer le potentiel $\varphi$ auquel correspondent les plus fortes valeurs de $\varphi_{\mathrm{s}}$ relevées : $\varphi \approx-11$, soit un potentiel d'environ 0,15 fois le potentiel du corps à une longueur de Debye de la surface. Ces estimations sont donc tout à fait satisfaisantes bien qu'on n'ose les qualifier de mesures.

3) Pour les polarisations positives, il arrive que le potentiel de la sonde flottante diminue brusquement au voisinage immédiat de la surface : nous avons montré en 3.1 que ceci caractérise un effet de sillage. Le phénomène est difficile à analyser dans le cas de la grille (cas des trous) mais il mérite une remarque dans le cas de la plaque.

Comme la plaque est rigoureusement parallèle au faisceau, ce manque d'ions ne provient pas d'un effet 
d'ombre mais d'une répulsion électrostatique ; cette observation ne porte en effet que sur la plus forte polarisation de plaque. Une estimation rapide montre qu'un champ de l'ordre de $1 \mathrm{~V} / \mathrm{cm}$ agissant sur quelques centimètres parvient à dévier le faisceau d'un millimètre environ. Il s'agit là d'une formation de gaine au sens classique du mécanisme, c'est-à-dire à cause de la répulsion exercée par la plaque sur une espèce chargée.

4) Pour les polarisations positives et mis à part l'effet précédent, les comportements de la sonde près de la plaque ou de la grille sont très différents.

Près de la grille, le potentiel de la sonde flottante reste constant, ce qui signifie d'après 3.1 que les effets d'absorption sont très faibles : la marge de variation du potentiel enregistrée étant inférieure à $4 \mathrm{mV}$, la relation (A.19) montre que le défaut de densité maximale est inférieur à $5 \%$. Il faut remarquer que cette valeur est plus petite que ce que donneraient des considérations géométriques au voisinage de la grille (soit $15 \%$ ) : un potentiel effectif [6] limite l'absorption des électrons par la grille.

Près de la plaque, le potentiel varie de façon continue ; les effets d'absorption sont donc bien observés. Nous n'avons pas tracé l'évolution correspondante de la densité maximale d'après la formule (A.19), mais on peut vérifier qu'on obtient un facteur de variation voisin de 0,5 pour les polarisations $+0,23,+1$ et $+1,4 \mathrm{~V}$. Dans le cas de la polarisation $+5 \mathrm{~V}$, la variation du potentiel flottant est supérieure à ce que prévoit la formule (A.20). C'est qu'il existe alors dans la fonction de distribution de la région entourant la sonde auxiliaire des exclusions de type balistique particulièrement importantes affectant les valeurs élevées de vitesses (Appendices I et II).

Nous devrons donc avoir des extensions de potentiel beaucoup plus grandes dans le cas de la plaque que dans le cas de la grille.

5.2 RÉSUltats OBTENUS AVEC LA SONDE ÉMISSIVE. - Les variations du potentiel autour de la plaque et de la grille sont représentées sur la figure 7. On constate que loin du corps (à $20 h$ environ) les gradients de potentiels sont presque nuls pour la grille mais encore importants pour la plaque. Cette expérience est la mise en évidence directe de l'extension des gaines des corps positifs en écoulement mésothermique, discutée en 2.1 et déjà signalée en [8].

Nous avons vérifié que pour les polarisations négatives de même amplitude, les gradients correspondants étaient au moins 10 fois plus faibles.

En absence de charges d'espace, le potentiel au voisinage de la plaque (sur $1 \mathrm{~cm}$ environ) décroîtrait linéairement. La présence d'une concavité sur les courbes de la figure 7 décèle donc la présence d'une charge d'espace négative au voisinage de la plaque. Mise à part la raréfaction éventuelle d'ions dans une couche mince signalée ci-dessus en A.3, nous avons vérifié à l'aide d'une sonde polarisée négativement que

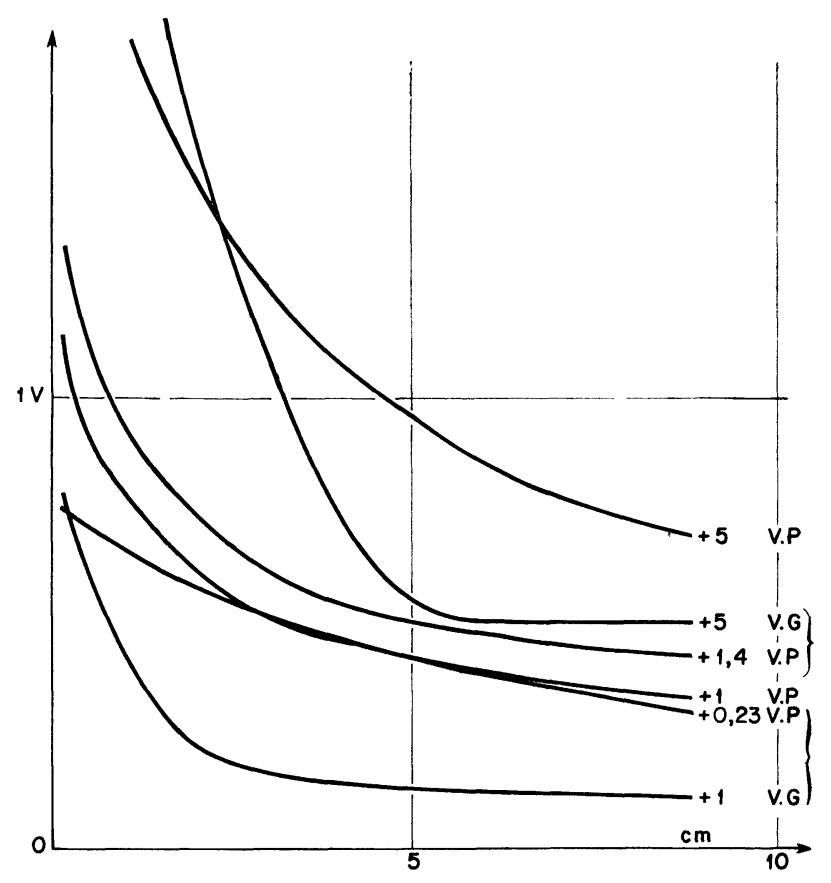

Fig. 7. - Potentiel flottant de la sonde émissive en fonction de sa distance à l'obstacle, grille $(G)$ ou plaque $(P)$, pour différentes polarisations. Les accolades indiquent des cas où les courants de grille et de plaque sont égaux. L'origine des ordonnées est arbitraire pour chaque cas.

la densité d'ions n'était pas modifiée au voisinage de la plaque. Il existe donc des concentrations d'électrons. Compte tenu des coefficients dus à l'absorption de l'ordre de 0,5 , observés en A.4, de telles concentrations ne s'expliquent qu'à l'aide de la formule tridimensionnelle (A.11) pour $\varphi>4$. Les courbes présentées sur la figure 7 concernent des potentiels de surface supérieurs à 10 (en valeurs réduites) ; les concentrations électroniques sont alors possibles. On remarque d'ailleurs que la courbure est plus faible pour les polarisations les moins fortes. Cette compréhension du phénomène à l'aide de la formule (A.11) implique à elle seule l'extension du champ à des distances très supérieures à la longueur de Debye, et même très supérieures à la plus grande dimension de l'obstacle fixe, ce qui souligne bien l'importance des perturbations apportées dans un plasma mésothermique par des corps positifs.

\section{Investigations du plasma autour de la sonde de} Sayers. - Les résultats suivants sont obtenus grâce à l'utilisation des sondes décrites en 3 et testées en 5 . Les conditions expérimentales étaient

$$
T_{\mathrm{e}}=600 \mathrm{~K}, \quad n=2 \times 10^{5} \mathrm{~cm}^{-3} \quad(h=4 \mathrm{~mm}) .
$$

Nous avons vérifié à l'aide de la sonde auxiliaire cylindrique flottante que le potentiel au milieu de la sonde de Sayers était bien négatif lorsque celle-ci était polarisée négativement. Ce qui suit concerne les polarisations positives de la sonde de Sayers.

6.1 Etude DE LA DENSITÉ ÉLECTRONIQUe. - Ces résultats proviennent de la sonde auxiliaire cylindrique 
flottante. La figure 8 et le tableau I montrent les valeurs obtenues pour des polarisations de la sonde de Sayers de $+1 \mathrm{~V}$ et $+5 \mathrm{~V}$ par rapport au caisson soit +21 et +94 en valeurs réduites et par rapport au plasma. Ces deux cas diffèrent sensiblement.

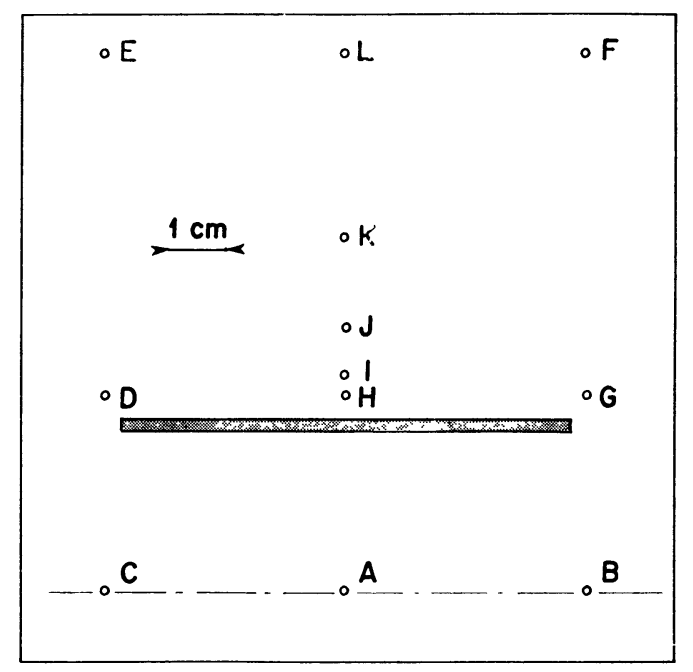

FIG. 8. - Emplacement des points où la densité électronique est donnée dans le tableau I. La vue de dessus d'une raquette de la sonde de Sayers est représentée ainsi que son plan de symétrie.

$\mathrm{A}+5 \mathrm{~V}$, il existe des modifications de courant d'ions ; ceci est certain pour les potentiels $\varphi_{\mathrm{s}}<\varphi_{\mathrm{s} \infty}$ où il $\mathrm{y}$ a appauvrissement en ions; ceci est possible au point $B$ où la valeur élevée du potentiel proviendrait en partie d'une augmentation de la densité ionique résultant de l'action de répulsion des grilles situées de part et d'autre.

$\mathrm{A}+1 \mathrm{~V}$, qui représente mieux les paramètres de fonctionnement de la sonde de Sayers, les perturbations ioniques n'apparaissent pas. Il faut toutefois remarquer que les plus petits rapports $n / n_{\infty}$ relevés $(0,69)$ sont plus faibles que pour la grille simple de même transparence étudiée en 5.1-4 (0,95).

On peut donc conclure que dans la région intéressant le fonctionnement d'une sonde de Sayers, la densité électronique pour les polarisations positives peut croître au-dessus de l'ambiante suivant la formule (A.11), mais être réduite, à cause de l'absorption, d'un facteur variable, avec la géométrie, l'orientation et la polarisation. Si l'on parvient à repérer le potentiel plasma, la cause de surestimation disparaît, de sorte que la sonde de Sayers doit donner des mesures par défaut de la densité : cette erreur peut atteindre environ $15 \%$ d'après une extrapolation au potentiel plasma des résultats rapportés sur le tableau I, alors que les expériences avec la grille |simple laissaient espérer $5 \%$.

6.2 Etude DU POTENTIEL. - A l'extérieur de la sonde de Sayers, les chutes de potentiel sont très voisines de celles représentées sur la figure 7 pour une grille simple. Au centre de la sonde, les effets des deux grilles s'ajoutent approximativement et on relève un potentiel égal à $20 \%$ environ de celui appliqué aux grilles. A $30 \mathrm{~cm}$ de la sonde de Sayers, il existe encore un champ électrique mesurable $(5 \mathrm{mV} / \mathrm{cm}$ à la polarisation $+1 \mathrm{~V})$.

Le satellite FR-1 comportant des cathodes émissives, qui sont nécessaires au fonctionnement des expériences embarquées, l'expérience décrite ci-dessus montre qu'il pouvait y avoir, pour ce satellite, une interaction directe entre la sonde de Sayers et les cathodes chaudes, bien que des précautions aient été prises à la fois pour éloigner ces éléments $(60 \mathrm{~cm})$ et pour tenter d'émettre les électrons en un faisceau orienté dans une direction qui les écartait de la sonde.

7. Conclusion. - Une étude précédente avait déjà signalé l'extension des gaines autour des corps positifs en écoulement mésothermique de plasma sans colljsions et donné un calcul numérique pour un cylindre [7], [8]. Le recours à des considérations tridimensionnelles a permis de comprendre pourquoi il se produit souvent une chute de potentiel importante au voisinage d'un corps positif bien que son champ s'étende toutefois à des distances très supérieures à la longueur de Debye. Nous avons mis en œuvre deux méthodes de mesure permettant l'une de suivre l'évolution du potentiel, l'autre de connaître le pourcentage de diminution de densité dû aux effets d'absorption.

\begin{tabular}{|c|c|c|c|c|c|c|c|c|c|c|c|c|c|}
\hline \multicolumn{2}{|c|}{$\begin{array}{c}\text { Localisation } \\
\text { d'après Fig. 8 }\end{array}$} & A & B & C & D & E & F & G & H & I & J & K & L \\
\hline \multirow{2}{*}{$+1 \mathrm{~V}$} & Potentiel (V) & $-0,324$ & $-0,330$ & $-0,313$ & $-0,313$ & $-0,324$ & $-0,334$ & $-0,62$ & $-0,324$ & $-0,327$ & $-0,327$ & $-0,327$ & $-0,327$ \\
\cline { 2 - 13 } & $\mathrm{n} / \mathrm{n}_{\infty}$ & 0,83 & 0,92 & 0,69 & 0,69 & 0,83 & 1 & & 0,83 & 0,89 & 0,89 & 0,89 & 0,89 \\
\hline \multirow{2}{*}{$+5 \mathrm{~V}$} & Potentie1 (V) & $-0,290$ & $-0,238$ & $-0,289$ & $-0,272$ & $-0,289$ & $-0,299$ & $-0,71$ & $-0,64$ & $-0,270$ & $-0,285$ & $-0,287$ & $-0,293$ \\
\cline { 2 - 12 } & $\mathrm{n} / \mathrm{n}_{\infty}$ & 0,72 & & 0,71 & 0,54 & 0,71 & 0,84 & & & 0,52 & 0,66 & 0,68 & 0,76 \\
\hline
\end{tabular}

TABleAU I

Densité électronique dans et autour de la sonde de Sayers, déduite du potentiel de la sonde auxiliaire cylindrique flottante, pour deux polarisations $(+1 \mathrm{~V}$ et $+5 \mathrm{~V})$. 
En ce qui concerne plus directement le fonctionnement de la sonde de Sayers, nous avons montré que le signal pouvait croître au-delà du potentiel plasma mais qu'un changement de pente devait être décelable dans le pire des cas (Fig. 2). Enfin, à moins que leur utilisation soit absolument imposée par ailleurs, les cathodes chaudes sont à éviter ; d'une part, leur présence interdit de prévoir par le calcul le potentiel de la sonde par rapport au plasma; d'autre part, les électrons émis sont susceptibles de perturber le plasma au voisinage de la sonde. Même si l'on parvient à repérer le potentiel plasma, la mesure conduira à une valeur par défaut de la densité électronique de 5 à $15 \%$ pour les grilles usuelles.

Remerciements. - Je remercie le Professeur W. P. Allis et le Docteur L. R. O. Storey de l'attention qu'ils ont prêtée au manuscrit. Cette étude a été partiellement financée par la Convention INAG-ONERA $\mathrm{n}^{\mathrm{0}}$ 070/150/0269.

\section{APPENDICE I}

Borne supérieure de la densité électronique dans une région positive. - En plus du vecteur position $\mathbf{x}$ nous utiliserons les grandeurs

$$
\mathbf{c}=\left(m / 2 k T_{\mathrm{e}}\right)^{1 / 2} \mathbf{w} \text { et } \varphi=-e V / k T_{\mathrm{e}},
$$

où w est le vecteur vitesse et $V$ le potentiel. Pour le régime stationnaire, l'équation de Vlasov s'écrit :

$$
\text { c. } \nabla_{x} f+\frac{1}{2} \nabla_{x} \varphi \cdot \nabla_{c} f=0 .
$$

On sait que cette éq. (A.2) est équivalente au système de ses équations caractéristiques :

$$
\mathrm{d} \mathbf{x} / \mathbf{c}=2 \mathrm{~d} \mathbf{c} / \nabla_{x} \varphi, \quad \mathrm{d} f=0,
$$

qui traduisent la conservation de $f$ le long d'une trajectoire de l'espace des phases $(\mathbf{x}, \mathbf{c})$.

Une solution de l'éq. (A.2) est :

$$
f=n_{\infty}\left(m / 2 \pi k T_{\mathrm{e}}\right)^{3 / 2} \exp \left(\varphi-c^{2}\right),
$$

qui est identique à (3) pour $\varphi=0$, c'est-à-dire le plasma ambiant. Lorsque $\varphi$ diffère de 0 , la fonction de distribution n'est donnée par (A.4) que sous réserve de conditions aux limites à préciser.

Nous faisons à ce propos un raisonnement de type « dans-hors » basé sur les éq. (A.3). Soit un point de position $\mathbf{x}$ dans la région où $\varphi \neq 0$; que vaut en ce point la fonction de distribution pour une vitesse $\mathbf{c}$ ? Il suffit de considérer la trajectoire aboutissant en $\mathbf{x}$ à la vitesse $\mathbf{c}$; ou bien :

a) elle provient du plasma ambiant ; à ce moment-là, la fonction de distribution est bien donnée par (A.4) car l'énergie est conservée le long de la trajectoire et la fonction de distribution est isotrope dans le plasma ambiant (donc ne dépend que de l'énergie);

b) elle provient d'un puits ou d'une source de particules, par exemple d'une surface absorbante (la fonc- tion de distribution correspondante est nulle) ou d'une surface émissive (la fonction de distribution est donnée par la loi d'émission de la surface) ;

c) elle ne provient ni du plasma ambiant ni d'un puits ni d'une source (particule piégée). Dans le cadre de l'hypothèse non collisionnelle, la fonction de distribution correspondante est nulle si l'on exclut toute autre cause de production d'électrons (rayonnement). Une discussion de l'existence des orbites fermées a été faite par Laframboise [6]. Une trajectoire est fermée soit parce que la vitesse c correspond à une énergie cinétique inférieure à l'énergie potentielle en $\mathbf{x}(c<\sqrt{\varphi})$ soit pour des raisons balistiques bien qu'on satisfasse la condition $c \geqslant \sqrt{\varphi}$. Cette condition

$$
c \geqslant \sqrt{\varphi}
$$

est donc nécessaire mais pas suffisante.

Supposons désormais qu'il n'y ait pas de surface émissive dans le plasma, ce qui réduit le cas $b$ ) à $f=0$. $\mathrm{La}$ fonction de distribution en un point de potentiel positif $\varphi$ est :

$\left\{\begin{array}{l}f=0 \text { pour } \quad c<\sqrt{\varphi} \\ f \text { donnée par (A.4) ou égale à } 0 \text { pour } c \geqslant \sqrt{\varphi} .\end{array}\right.$

En prenant $f$ donnée par (A.4) pour tout $c \geqslant \sqrt{ } \bar{\varphi}$, nous obtiendrons une borne supérieure de la densité électronique. Nous allons calculer cețte valeur dans trois hypothèses.

a) Cas monodimensionnel : une seule composante est affectée par la condition (A.6) et l'on obtient la formule :

$$
n \leqslant n_{\infty} \exp \varphi \operatorname{erf} c \sqrt{\varphi}
$$

où $\operatorname{erf} c \sqrt{\varphi}=1-\operatorname{erf} \sqrt{\varphi}$, erf étant la fonction erreur.

Pour

$$
\varphi \rightarrow 0, \quad n \leqslant n_{\infty}(1-2 \sqrt{\varphi} / \sqrt{\pi})
$$

et pour

$$
\varphi \rightarrow \infty, \quad n \leqslant n_{\infty} / \sqrt{\pi \varphi} .
$$

La représentation complète de la formule (A.7) est donnée sur la figure 1.

b) Cas bidimensionnel : il s'agit d'une géométrie cylindrique quelconque où la composante de vitesse $c_{z}$ est supposée non modifiée par le potentiel. Comme les conditions (A.6) ne portent que sur le module de $\left(c_{x}, c_{y}\right)$, on peut raisonner sur la distribution cylindrique

$$
f=n_{\infty} \sqrt{m / 2 k T_{\mathrm{e}}} 2 c \exp \left(\varphi-c^{2}\right),
$$

avec

$$
c^{2}=c_{x}^{2}+c_{y}^{2} \geqslant \varphi
$$

d'où :

$$
n \leqslant n_{\infty}
$$


c) Cas tridimensionnel général : les trois composantes $c_{x}, c_{y}$ et $c_{z}$ sont affectées par le potentiel. Mais comme les conditions (A.6) ne portent que sur le module, on peut raisonner sur la distribution sphérique

$$
f=n_{\infty} \sqrt{m / 2 \pi k T_{\mathrm{e}}} 4 c^{2} \exp \left(\varphi-c^{2}\right)
$$

d'où

$$
n \leqslant n_{\infty}(\exp \varphi \operatorname{erf} c \sqrt{\varphi}+2 \sqrt{\varphi} / \sqrt{\pi}) .
$$

Pour $\varphi \rightarrow 0, n \leqslant n_{\infty}(1+\varphi)$.

Pour $\varphi \rightarrow \infty, \quad n \leqslant 2 n_{\infty} \sqrt{\varphi} / \sqrt{\pi}$.

La représentation complète de la formule (A.11) est donnée sur la figure 1 .

Dans le cas monodimensionnel $a$ ) la condition (A.5) est suffisante en absence d'absorption; en présence d'une surface absorbante plane, la densité est simplement divisée par 2.

Dans les cas pluridimensionnels $b$ ) et $c$ ) des valeurs exactes de $n$ pourraient être obtenues numériquement (voir [6] dans le cas de champs avec symétries ou [7] sans symétries).

\section{APPENDICE II}

Relations entre le potentiel flottant de la sonde auxiliaire et les paramètres du plasma. - 1) ConfiguRATION MONODIMENSIONNELLE. - Nous ne nous intéressons qu'à la composante de courant sur la sonde dans la direction où le potentiel varie. Ce courant est proportionnel à

$$
j=\exp \varphi_{\mathrm{s}} \int_{c_{1}}^{\infty} c \exp \left(-c^{2}\right) \mathrm{d} c .
$$

La borne d'intégration $c_{1}$ dépend du plasma environnant au potentiel $\varphi$. En se limitant au cas $\varphi \geqslant 0$, et en remarquant que dans le plasma ambiant $(\varphi=0)$, la sonde a un potentiel $\varphi_{\mathrm{s} \infty}$ négatif limitant le courant d'électrons à une valeur qui doit rester égale au courant d'ions, on voit que, par continuité, la relation

$$
\varphi_{\mathrm{s}}<0 \leqslant \varphi
$$

sera partout satisfaite et que

$$
j=\exp \varphi_{\mathrm{s}}=\exp \varphi_{\mathrm{s} \infty}
$$

entraîne :

$$
\varphi_{\mathrm{s}}=\varphi_{\mathrm{s} \infty} .
$$

Le potentiel flottant de la sonde auxiliaire est donc indépendant du plasma environnant. Ce résultat, négatif quant à la possibilité d'utiliser cette sonde pour le diagnostic, est une introduction à ce qui suit et constitue une mise en garde importante.

2) Configuration Bidimensionnelle. - La sonde auxiliaire est supposée alignée avec la dimension négligée. D'après l'appendice I la fonction de distribution à prendre en considération est :

$$
f=\alpha n_{\infty} \sqrt{m / 2 k T_{\mathrm{e}}} 2 c \exp \left(\varphi-c^{2}\right),
$$

la fonction $\alpha$ valant 0 ou 1 suivant qu'il existe ou non des exclusions pour des raisons balistiques ou à cause de surfaces absorbantes. Commençons par ne tenir compte que des exclusions du type (A.5) liées à la conservation de l'énergie en potentiels positifs. Le courant d'électrons sur la sonde auxiliaire est proportionnel à :

$$
j=\frac{2}{\sqrt{\pi}} \exp \varphi_{\mathrm{s}} \int_{c_{1}}^{\infty} 2 c^{2} \exp \left(-c^{2}\right) \mathrm{d} c .
$$

La borne $c_{1}$ dépend de la configuration du potentiel. Il suffit de remarquer qu'un potentiel négatif $(\varphi<0)$ constitue une barrière et que $c_{1}$, qui correspond à l'énergie minimale des électrons reçus, est différent de zéro dès que $\varphi_{\mathrm{s}}$ est supérieur à $\varphi$ ou à 0 ; on a alors respectivement $c_{1}=\sqrt{\varphi_{\mathrm{s}}-\varphi}$ ou $c_{1}=\sqrt{\varphi_{\mathrm{s}}}$, d'où les valeurs suivantes de $j$ :

$$
\begin{gathered}
j=\exp \varphi_{\mathrm{s}} \text { pour } \varphi_{\mathrm{s}}<\varphi<0 \quad \text { ou } \varphi_{\mathrm{s}}<0<\varphi, \\
j=\exp \varphi_{\mathrm{s}}\left[\frac{2}{\sqrt{\pi}} \sqrt{\varphi_{\mathrm{s}}-\varphi} \exp \left(\varphi-\varphi_{\mathrm{s}}\right)+\operatorname{erf} c \sqrt{\varphi_{\mathrm{s}}-\varphi}\right] \\
\text { pour } \begin{array}{l}
\varphi<\varphi_{\mathrm{s}}<0 \\
\varphi<0<\varphi_{\mathrm{s}}
\end{array} \\
j=\exp \varphi_{\mathrm{s}}\left[\frac{2}{\sqrt{\pi}} \sqrt{\varphi_{\mathrm{s}}} \exp \left(-\varphi_{\mathrm{s}}\right)+\operatorname{erf} c \sqrt{\varphi_{\mathrm{s}}}\right] \\
\text { pour } \begin{array}{l}
0<\varphi_{\mathrm{s}}<\varphi \\
0<\varphi<\varphi_{\mathrm{s}} .
\end{array}
\end{gathered}
$$

Ces trois fonctions sont représentées sur la figure 3.

Dans le plasma ambiant où $\varphi=0$, le potentiel flottant de la sonde auxiliaire est $\varphi_{\mathrm{s} \infty}<0$. Si la sonde auxiliaire est déplacée vers une région perturbée positive, la première relation (A.16) s'applique toujours par continuité, ce qui élimine en pratique l'utilisation de la troisième relation (A.16). Si la sonde auxiliaire est déplacée vers une région négative, la première relation (A.16) s'applique tant que $\varphi \geqslant \varphi_{\mathrm{s} \infty}$; pour $\varphi<\varphi_{\mathrm{s} \infty}$, il faut utiliser la deuxième relation (A.16). La conservation du courant d'électrons s'écrit :

$$
\begin{aligned}
\exp \varphi_{\mathrm{s}}\left[\frac{2}{\sqrt{\pi}} \sqrt{\varphi_{\mathrm{s}}-\varphi} \exp \left(\varphi-\varphi_{\mathrm{s}}\right)\right. & \left.+\operatorname{erf} c \sqrt{\varphi_{\mathrm{s}}-\varphi}\right]= \\
& =\exp \varphi_{\mathrm{s} \infty} .
\end{aligned}
$$

La relation (A.17) permet de suivre l'évolution du potentiel local pour $\varphi<\varphi_{\mathrm{s} \infty}$ et la figure 3 montre que $\varphi_{\mathrm{s}}$ croît dans ce cas. Lorsque $\varphi_{\mathrm{s}}-\varphi>1,5$, la relation (A.17) s'écrit :

$$
\varphi_{\mathrm{s}}-\varphi \approx \frac{\pi}{4} \exp 2\left(\varphi_{\mathrm{s} \infty}-\varphi\right) .
$$


Cette équation montre que pour $\varphi<\varphi_{\mathrm{s} s o}$, le potentiel flottant de la sonde auxiliaire atteint rapidement des valeurs très positives : ces situations sont incompatibles avec l'hypothèse des faibles perturbations dues à la sonde et nous limitent alors à des conclusions qualitatives. Résumons :

- pour $\varphi \geqslant \varphi_{\mathrm{s} \infty}$, le potentiel $\varphi_{\mathrm{s}}$ est insensible aux variations de $\varphi$;

- pour $\varphi<\varphi_{\mathrm{s} \infty}, \varphi_{\mathrm{s}}$ peut atteindre des valeurs fortement positives donnant une estimation grossière du potentiel $\varphi$ pour un volume étendu $(\gg h)$ à l'aide de la relation (A.18). On note qu'en aucun cas, l'évolution de $\varphi_{\mathrm{s}}$ ne suit directement l'évolution de $\varphi$. Désormais nous ne considérons que l'éventualité $\varphi \geqslant \varphi_{\mathrm{s} \infty}$ qui nous ramène à la première relation (A.16). Cette relation $j=\exp \varphi_{\mathrm{s}}$ est une borne supérieure du courant car on a pris $\alpha=1$ dans l'éq. (A.14) pour toute vitesse $c$.

Il est clair que les conditions balistiques (absorption exclue) qui imposent $\alpha=0$ concernent les plus petites valeurs de $c$ au niveau du potentiel $\varphi$. Au niveau de la sonde répulsive, ces exclusions peuvent donc ne pas apparaître. L'intégration donnant le courant (A.16) sera donc beaucoup moins affectée par ces exclusions que l'intégration donnant la densité (A.9) : la sonde auxiliaire est donc peu apte à suivre des diminutions de densité électronique liées à ce phénomène. Par contre si $\alpha=0$ à cause d'un effet d'absorption, la perturbation peut être suffisante pour provoquer une modification sensible du potentiel flottant de la sonde auxiliaire. Pour expliciter, nous supposons que les effets d'absorption sont cause que $\alpha=0$ pour certaines directions de vitesse indépendamment du module. Les écarts relatifs sur le courant et la densité sont alors égaux. Les relations (A.9) et (A.16) conduisent à :

$$
n=n_{\infty} \exp \left(\varphi_{\mathrm{s} \infty}-\varphi_{\mathrm{s}}\right) .
$$

On peut donc déduire la densité électronique locale à partir du potentiel flottant de la sonde auxiliaire cylindrique quand $\varphi \geqslant \varphi_{\mathrm{s} \infty \infty}$. Au voisinage immédiat d'une paroi $n=0,5 n_{\infty}$; on peut donc prévoir que $\varphi_{\mathrm{s}}$ variera dans l'intervalle

$$
\varphi_{\mathrm{s} \infty} \leqslant \varphi_{\mathrm{s}} \leqslant \varphi_{\mathrm{s} \infty}+0,69 .
$$

3) Configuration tridimensionnelle. - Comme la sonde auxiliaire est cylindrique, les exclusions dues au potentiel $\varphi$ du plasma environnant conduiront aux mêmes conclusions qu'au paragraphe 2). De même, les exclusions de type balistique n'affectent pas la sonde qui ne décèle que les effets d'absorption à condition qu'ils correspondent à des directions faisant un angle suffisamment grand avec son axe. A cette condition, on retrouve donc toutes les conditions du paragraphe 2). L'utilisation d'une sonde auxiliaire sphérique ne présenterait donc aucun intérêt supplémentaire.

\section{Bibliographie}

[1] McKenzie, E. C., Sayers, J., Planet. Space Sci. 14 (1960) 731.

[2] Baguette, J. M., GRI/NT/76, 1968.

[3] Sayers, J., Wilson, J. W. G., Loftus, B., Proc. R. Soc. A 311 (1969) 501.

[4] Sturges, D. J., Communication privée à R. Godard.

[5] Goodall, C. V., Planet. Space Sci. 19 (1971) 827.
[6] LAFRAmboise, J. G., UTIAS Report n 100, 1966.

[7] Fournier, G., Publication ONERA n ${ }^{\circ}$ 137, 1971.

[8] Taillet, J., Brunet, A., Fournier, G., Conférence IUTAM sur la Dynamique des gaz ionisés, Tokyo, 13-18 septembre 1971.

[9] Pigache, D., AIAA 4th Fluids and plasma dynamics conference, 21-23 juin 1971, AIAA Paper n 71-608. 